\title{
Effect of folic acid supplementation during pregnancy on brain health of the child at 11 years: the FASSTT Offspring trial
}

\author{
Aoife Caffrey ${ }^{1}$, Kristina Pentieva ${ }^{1}$, Helene McNulty ${ }^{1}$, Pramod Gaur ${ }^{2}$, Joel B. Talcott ${ }^{3}$, \\ Caroline Witton ${ }^{3}$, Anthony Cassidy ${ }^{4}$, Marian McLaughlin ${ }^{4}$, Diane J. Lees-Murdock ${ }^{5}$, \\ Rachelle E. Irwin ${ }^{5}$, Colum P. Walsh ${ }^{5}$ and Girijesh Prasad ${ }^{2}$ \\ ${ }^{1}$ Nutrition Innovation Centre for Food and Health (NICHE), Ulster University, Coleraine, United Kingdom, \\ ${ }^{2}$ Intelligent Systems Research Centre, Ulster University, Derry Londonderry, United Kingdom, \\ ${ }^{3}$ Aston Brain Centre, Aston University, Birmingham, United Kingdom, \\ ${ }^{4}$ Psychology Research Institute, Ulster University, Coleraine, United Kingdom and \\ ${ }^{5}$ Genomic Medicine Research Group, Ulster University, Coleraine, United Kingdom
}

\begin{abstract}
Periconceptional folic acid (FA) has an established role in the prevention of neural tube defects (NTDs), leading to global recommendations for FA supplementation before and in early pregnancy. However, it is unclear whether there are any benefits for offspring brain health arising from continued maternal FA supplementation beyond the first trimester. The aim of this study was to investigate the role of maternal folate nutrition during pregnancy in relation to cognitive performance and brain function in the offspring at 11 years. The children of mothers who had participated in a randomised trial of Folic Acid Supplementation in the Second and Third Trimesters (FASSTT) were investigated, providing a unique opportunity to examine offspring brain health in relation to maternal folate (the FASSTT Offspring trial; $n=68$ ). Cognitive performance was assessed using the Wechsler Intelligence Scale for Children, Fourth UK Edition (WISC-IV). The WISC-IV measures Full Scale IQ and specific domains of cognitive performance: Verbal Comprehension, Perceptual Reasoning, Working Memory and Processing Speed. Brain function was measured using magnetoencephalography (MEG) in a subset of the child participants $(n=33)$. The results showed no significant difference in Full Scale IQ between the children of mothers who had received folic acid versus placebo during pregnancy $(P=0.993)$. Processing Speed subtest scores were however significantly higher in the folic acid group compared with placebo (Symbol Search: $P=0.046$ and Cancellation: $P=0.011)$. The application of MEG analysis showed that at rest, there were differences in brain functioning with significantly lower overall power at Broad band $[1-48 \mathrm{~Hz}](P=0.041)$ and a trend (not significant) towards lower power in all other frequency bands (Theta, Mu, Beta, Low Gamma and High Gamma) in children from the FA group compared with placebo. Results for the responses to the language task (congruent and incongruent sentences) in children from the FA group showed significantly lower power within the Theta band $[4-8 \mathrm{~Hz}]$ and significantly higher power within high frequency bands i.e. Beta [13-30 Hz] and High Gamma [49-70 Hz]. This suggested more efficient language processing abilities in these children compared to children of mothers in the placebo group. The findings provide scientific evidence that continuing FA supplementation beyond the periconceptional period that is protective against NTDs, may be beneficial for brain health in the offspring. Furthermore, this study demonstrates that MEG is a useful tool for objective assessment of functional brain activity in healthy children in response to nutrition intervention.
\end{abstract}

\section{Conflict of Interest}

There is no conflict of interest 